\title{
Researching of the Three-Dimensional Virtual Simulation Campus Scene's Construction Technology
}

\author{
Li Wang*
}

Department of education science and technology, Shanxi Datong University, Datong, China

\begin{abstract}
With the development of network technology, many colleges and universities have established a campus network to achieve publicity of their campus. According to the current situation, the majority of the universities show their styles just through words and two-dimensional image, which received poor results. To strengthen the propaganda of the school, improve the overall image, improve the enrollment and employment and lay the foundation for wisdom university, this paper is based on real campus and uses virtual technology to build a three-dimensional virtual simulation campus, so that users can understand the school and experience a real sense of immersing in the situation. The article deeply discusses the meaning of the three-dimensional virtual campus, the design process, related technology and scene building process, in order to play a valuable role in constructing large-scale three-dimensional scene.
\end{abstract}

Keywords: Modeling, three-dimensional scene, virtual campus.

\section{INTRODUCTION}

Virtual reality technology is an integrated technology, which uses computer graphics, human-computer interaction technology, sensor technology, artificial intelligence and other technical means to enable users with necessary means and computers naturally to experience and interact with a computer and form the realistic three-dimensional vision, such as vision, hearing, touch reception, etc. [1].

Three-dimensional virtual simulation campus is digital, and three-dimensional, which reappears the campus scene through virtual reality technology. Users can browse and roam the inner and external campus through the keyboard, mouse and other input devices, understanding the campus environment with proximity.

Virtual Campus can increase schools' popularity, realize good foreign propaganda and make it easy for users to understand the school, which is the basis of the construction of the digital campus. Its research and building have important significance to the latter construction of digital campus and wisdom campus.

Virtual campus system mainly consists of two parts, namely the construction of the virtual campus and design of interactive Virtual Tour. In this paper, the author makes Shanxi Datong University virtual campus as an example, discussing the three-dimensional virtual scene of the design process, related technology and building process.

\section{THE DESIGN PROCESS AND RELATED TECH- NOLOGIES OF THE VIRTUAL CAMPUS SCENE}

The production of virtual campus scene is more complex, involving many related technologies.
When we are making the virtual scene, we should firstly use the appropriate graphics software to draw the entire campus plan according to the actual size of the campus buildings to determine the layout and location of each building. In the construction industry, the most popular floor plan drawing software is AutoCAD, which is easy to use and powerful.

After you draw a good plan, you can use threedimensional modeling tool to create the entire threedimensional virtual campus scene. Common tools for 3D modeling conclude 3ds Max, MAYA. 3ds Max is functional and easy to use, with a variety of modeling methods, especially the polygon modeling is fit for architectural modeling. MAYA has a strong curve modeling tool, which is well suited for character modeling and complex animation. $3 \mathrm{ds}$ Max is usually used for modeling the virtual campus.

After the accomplishment of modeling the threedimensional virtual simulation, we need to give material to the well-accomplished models to accurately express the model's color, texture, light reflection, refraction and other physical properties. In order to reflect the information of surface texture, we need to generate maps. Map generation primarily use your camcorder or digital camera to get the building's surface texture image, then use Photoshop software to amend or edit generated maps appropriately. In addition to using the map, we need to use 3ds Max Material to reflect the other physical properties such as the reflection of the light and the refraction.

After giving the model material, we needs to add lighting effects to simulate real-world's light to make the scene wellarranged, more real and natural. In order to reduce the computation time of the CPU, we can use map baking technology to turn light information into map form.

Finally, since each scene have been finished, we need to place each model in the corresponding position according to CAD plane underlay drawn. Then we should sort various 


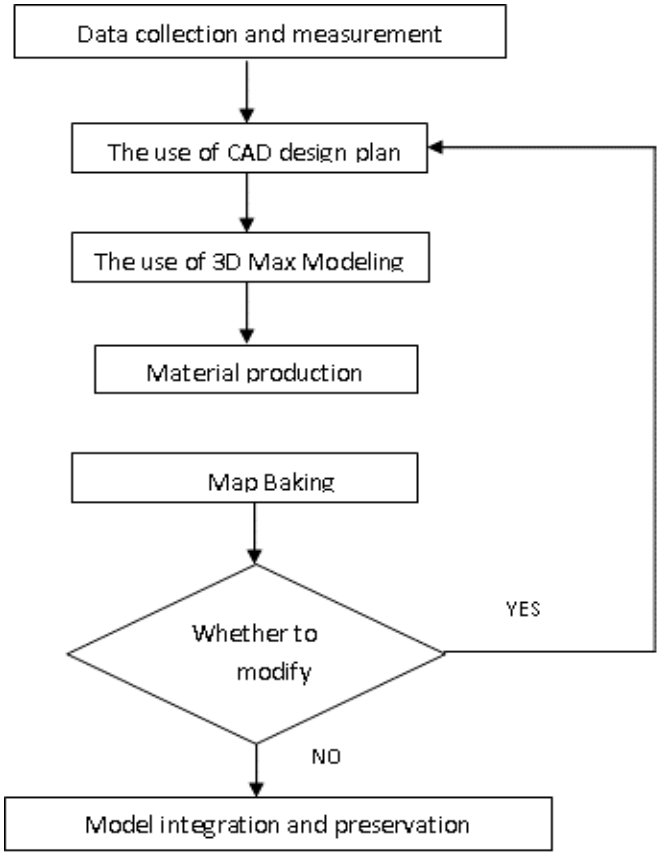

Fig. (1). The design process of the virtual campus.

scenes into a complete virtual campus. The specific design of virtual campus is shown in Fig. (1).

\section{THE PROCESS OF THE CONSTRUCTION OF THE VIRTUAL CAMPUS' THREE-DIMENSIONAL SCENE}

The following is three-dimensional virtual simulation of Shanxi Datong University campus, which can illustrate the process of building a virtual three-dimensional scene.

\subsection{Draw a Plan}

Firstly, draw an overall plan of Shanxi Datong University by using AutoCAD according to historical data and actual measurement data. We have mastered before to determine layout and location of pavements and buildings. Controlling campus buildings macroscopically can lay the foundation for the later integration of the entire campus scene.

Make the North Campus of Datong University as an example, CAD drawing floor plan is as following:
1. Create an overall plan of North Campus.

2. Draw roads and all kinds of controlling limits, red lines and so on. In the process of drawing lines, straight lines, arcs, and their move operations are mainly used. North Campus' good road map is shown in Fig. (2).

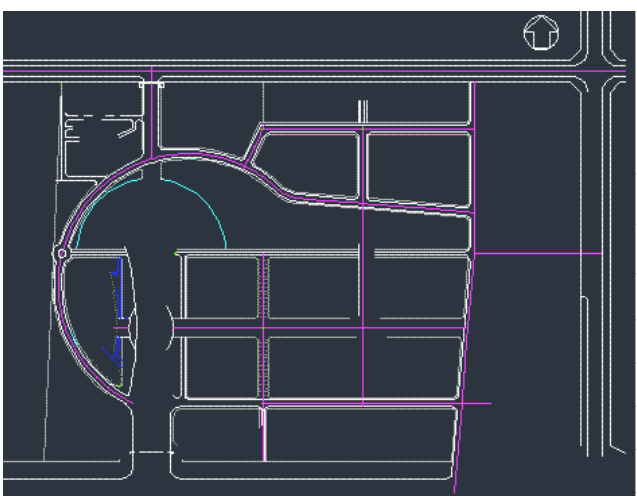

Fig. (2). North Campus Road Map.

3. According to the actual situation draw the outer contour of the building. As you draw the North student dormitory, the outer contour of the building are straight lines. According to the actual size to determine the length of each segment, draw the position of doors and windows and mark various size, elevation, coordinates, and text. North Dormitory's CAD picture is shown in Fig. (3).

4. According to the method described above, design the CAD base map of all the buildings on the North Campus. Put the designed CAD drawings in the road map of the North, thus getting the overall CAD drawing of the North Campus.

\subsection{Modeling}

After drawing CAD drawings, you can use the powerful $3 \mathrm{ds}$ Max modeling tool for modeling a campus building. $3 \mathrm{ds}$ Max includes a variety of modeling methods. How to choose the appropriate modeling approach is the key to modeling.

\subsubsection{Select Modeling Method}

3 ds Max that can be used to model all campus buildings is rich in modeling capabilities. Different objects have different modeling methods, the same object can have a variety of

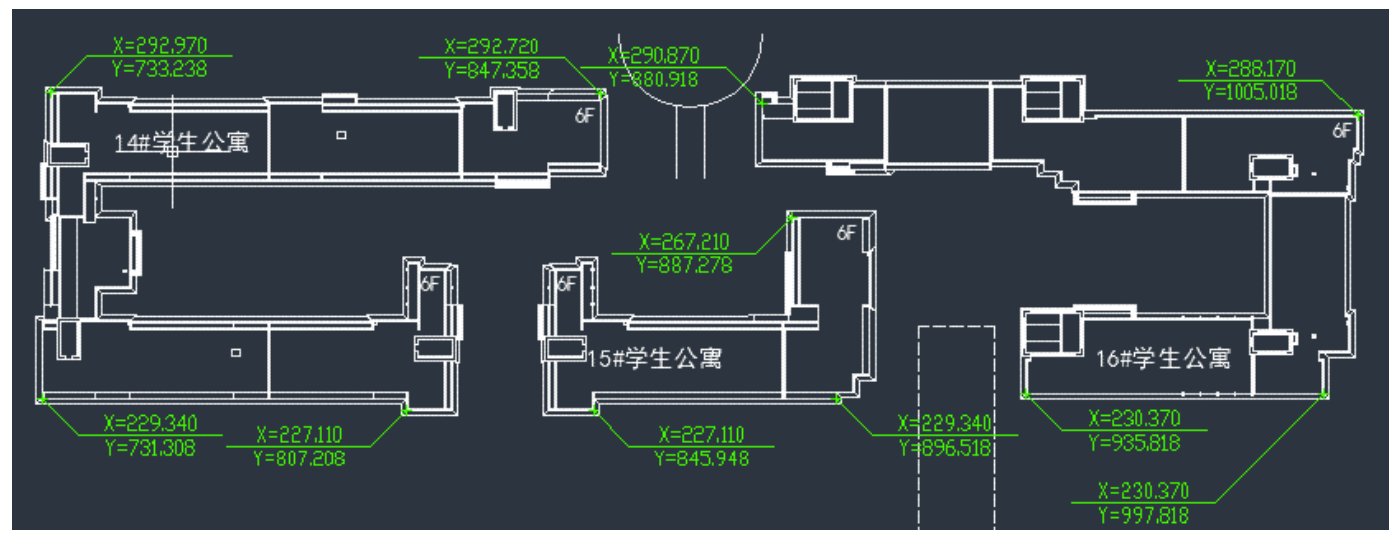

Fig. (3). North Dormitories CAD Drawings. 


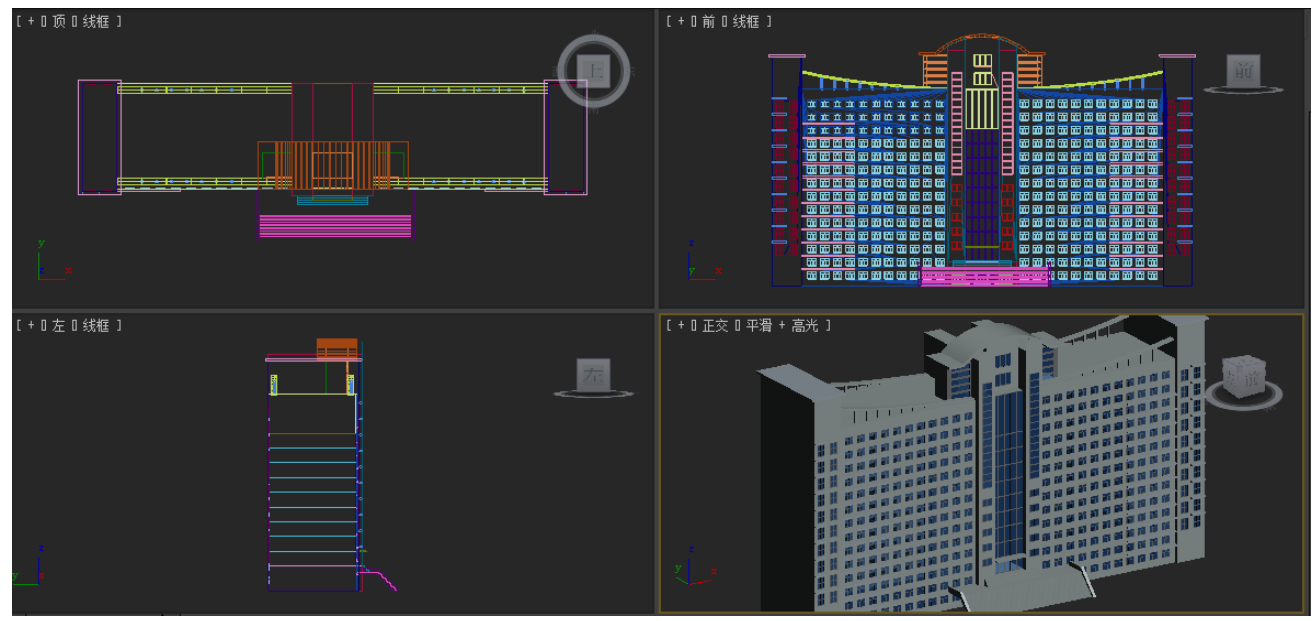

Fig. (4). 3D model rendering of the North Complex Building.

modeling methods. Common modeling methods are described below.

1. For geometry and expansion of geometry and combined objects of each other, we can make models directly. Such as the common table and chairs in our daily life.

2. For the complicated subjects that need a standard geometry or patulous aggregation, you need to convert its initial shape into "Editable Polygon" or "Editable Nets" and select the points and planes in the editing panel to achieve the ideal effect. For example, in the modeling of the computer screen, we use rectangles of basic geometry as the initial form, and converts it to "Editable Poly", then select "Polygon" level, select which parts should be stretched. This method can also be used to make models for subjects whose surface is uneven, such as keyboard, television, mobile phones and keys on the phone.

3. For some objects which can not be directly made for models, such as vase, we can use two-dimensional modeling to draw the side acres, and make models by lathe modifier.

4. For some irregular objects which have a variety of crosssection, loft-modeling can be selected. Draw its lofting path and lofting shape, and set the appropriate lofting steps percentage, then make a model, such as computer monitor's modeling.

5. For three-dimensional character's modeling, we can select polygonal modeling by combining sheet with netting which can be achieved by points' weaving nets. That is to say, many facets and slices are connected according to the actual need, constituting the final model.

6. For the models that need consolidation, intersection and remaining of two or more objects, we can use geometry Boolean operations to make models. Boolean operations are included in the intersection, difference, amalgamation. It can be designed according to the actual needs, such as hollowing out the windows on the wall can be modeled by a differential set.

7. For those that use conventional modeling methods resulting in too much of points, we can use a map modeling and other methods in actual modeling. Map modeling is used for plants and some distant models that do not need more. As for modeling the keyboard in the engine room, if we use Boolean operations, we will face several models with too many facets. Using a box to make a model and adding texture on the surface of the model will significantly reduce the number of faces.

If the the same model can be completed by a variety of modeling methods, the modeling methods of least faces should be used. When modeling distant objects, we can appropriately reduce the "fragment" to reduce the number of faces.

\subsubsection{Modeling Process}

We make the North Complex Building of Shanxi Datong University as an example to illustrate the specific modeling of buildings. After modeling, the final rendering of the North Complex Building of Shanxi Datong University is shown in Fig. (4).

Firstly, before modeling we should analyze the complex building structures. On the whole, the building can be divided into six parts, the outer layer of the hall, the left the house, the middle part, the top, right side of the House, front stairs, while the left side body and the right side of the House floor the same, so long as we complete one side of the model, then through the mirror copy other side can be obtained.

\section{Modeling of complex building}

1) In 3ds Max display units and system units are set to meters. Build a cuboid called Box01 measuring $9.5 \mathrm{~m}$ long, $7 \mathrm{~m}$ wide, $2.5 \mathrm{~m}$ high as the wall of the hall floor in the perspective.

2) Convert Box01 into editable polygon. Remove the front part of the remaining parts as the hall door, removed part were black.

3) In the new view, build the plan01 measuring $2.5 \mathrm{~m}$ long, $7 \mathrm{~m}$ wide in the black part of box01. Build a new cuboid of the size and location with the left door, and make it through Plan01.

4) Select Box02, and choose "compound objects" in the geometry drop-down box, then select "Boolean" button in the options that provided. Select "difference set (B- 
A)" in "Boolean" drop-down box, then select "Get operand B", and move the mouse to the plane Plane01. After the operation is completed, the plane will appear a hollow of the same size with Box02 on Plan01.

5) Determine the positions of the two doors by the above method.

6) Next is the door frame's production. In reality, the surface of an aluminum door's frame generally has some projections. But in the process of modeling Shanxi Datong University's outside buildings, since the door is small compared with the entire virtual campus, it the details can be ignored. We can make the frame of the door by rectangular and use the move tool and alignment tools to do alignment operation. After the framework models is built, all rectangular frames should be selected and all of them should be named "mengkuang1". Make a New plane Plan02 and give it glass material, align it with the frame by aligning tool.

7) Make the other two frames and glasses by the same method, and all the objects should be aligned by the proper alignment. All selected objects should be named "yicengdating". The accomplished comprehensive the first floor's hall of the North Complex Building has effects shown in Fig. (5).

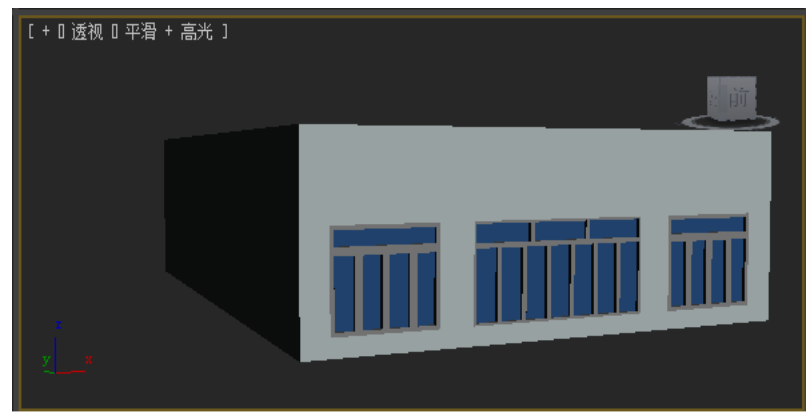

Fig. (5). The first floor's hall of the North Complex Building.

2. Next, complete the left side, the middle part, the top, right side of the House, front steps of Comprehensive Building. Then integrate these six parts as physical integration of these six parts to give a final image of the building.

\subsection{Material Production}

In order to make the model to achieve the simulation results, we need real object as the base material to the give the model material after the establishment of a good model. Material determines the surface properties of objects. Simply to say, a material is what the texture of objects appears to be. It is a combination of materials and textures of objects. In rendering program, the material is a comprehensive visual attributes of objects surface. These attributes are the surface texture, color, transparency, smoothness, luminosity, reflectivity, refractive index, etc. [2].

\subsubsection{Preparation of Material Library}

You need to use a professional camera or video camera to take photos of campus' objects before making materials. Due to the difference between shooting positions and ligh- ting, photographs and original scenery there may have some discrepancy, then we need to use Photoshop software to make proper adjustments and modifications, and store these in JPG format into the material library, for making material.

\subsubsection{VRay Renderer Parameter Settings}

In material production, use VRay as the default renderer. Before using VRay renderer, corresponding parameters need to be set.

\subsubsection{Material Production Process}

Material production process is more complex, a material often needs to go through slight adjustment to achieve the desired results. In practice, we must give them a material after finishing a model. Doing so not only can avoid omissions, but also can improve efficiency, such as the production of Datong University Library Reading Room, since there are multiple same chair in the scene, then you can add the material of a chair, and copy the chair to complete the production of all the chairs in the scene.

Take Datong University Library Reading Room tables and chairs for example, and describes the production process of VRay wood-based materials. Specific steps are as follows:

1. Select the reading room desk's surface in perspective, then press M button to open the Material Editor.

2. Click "Get Material" button in the pop-up "Material/Map Browser" dialog box, double-click the "VrayMtl" option.

3. Choose a material sphere that should be named as "zhuomian". Set the material spheres as follows: diffuse is a grainy textures; reflection is 40 ; high gloss is 0.65 ; reflective gloss is 0.8 ; bump value associated with wood material is $25 \%$.

4. Click "Assign Material To Selected Objects Button" to put the adjusted the material to wood library reading room desktop.

5. Render the scene, contradistinction pictures of adding a contrast material or not are shown in Fig. (6).
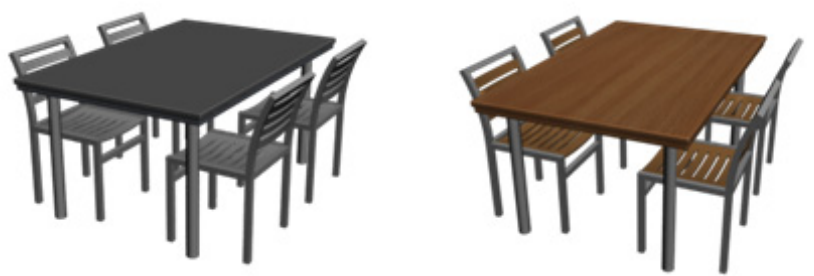

Fig. (6). Chairs give the material before and after comparison chart.

\subsection{Baking The Scene}

We can simulate reality campus' lighting effects by texture baking technology or lighting technology. When using lighting technology, CPU will perform a lot of calculations. The rendering speed is slow. The texture baking technology can render the lighting information in the $3 \mathrm{ds}$ Max into a map, then paste the map into the scene to simulate light [3]. Using the map baking technology to achieve light simulation can reduce CPU operation time and improve the rendering speed. 


\subsection{Integration Scene Models}

After each scene finished, put each model in the corresponding position and integrate the various scenes into a complete virtual campus according to the CAD plane drawn before. After the construction of three-dimensional scene of virtual campus, it can be imported to make a design of a virtual interactive tour. If the virtual scene is too large, it can not be integrated in $3 \mathrm{ds}$ Max, the entire scene can be divided into various independent sub-sections. Lead each sub-section sand the campus CAD plane respectively into the virtual interactive platform. Integrate all of these in the virtual interaction platform.

\section{CONCLUSION}

In the whole process of building a three-dimensional virtual simulation campus scene, the most critical issue is the faces number of the model, which relates to the normal operation of the three-dimensional virtual campus system. Therefore, it is necessary to strictly control the the number of faces in the construction of the scene, especially the modeling, the process of making materials and lighting. It should not only ensure the user's visual needs, but also ensure the least number of the surface.

\section{CONFLICT OF INTEREST}

The author confirms that this article content has no conflict of interest.

\section{ACKNOWLEDGEMENTS}

This article belongs to Shanxi Province Education Science "The $12^{\text {th }}$ five-year planning project" based on the stage research of "Researching of the $3 \mathrm{~d}$ virtual campus navigation simulation system's construction technology based on Unity3D" (item number: GH-13092) phase of the study results, and get the founding of Shanxi Datong University Youth Project "Design and implementation of Shanxi Datong University virtual campus roaming system based on Virtools" (item number: 2013Q1).

\section{REFERENCES}

[1] Y.F. Ma, Y.T. Xue and H.S. Nan.Virtual reality technology and its application[M]. Beijing: China Railway Press, 2011:2-2.

[2] Information on http://baike.baidu.com.

[3] Information on http://wenku.baidu.com

[4] X.Y. Huang, M. Du, F.F. Dong and M.H. Guo. 3ds Max 2011 standard tutorial. Beijing: Tsinghua University Press. 2011.

[5] Y. Fan, M. Yang and W.C. Sun. 3ds Max three dimensional animation production base and operating instructions. Beijing: Tsinghua University Press. 2010. 